\title{
Design and Implementation of Cascade LQR Controller for Stabilization of Two-Wheeled Robot
}

\author{
Ts. Slavov, J. Kralev, P. Petkov
}

Key Words: Two-wheeled robot control; LQR control; embedded systems.

Abstract. In this paper the developed two-wheeled robot and cascade LQR controller, Kalman filters, PI and PID controllers are presented. The cascade LQR controller stabilizes the two-wheeled robot in upright position. The PID controller ensures good tracking of wheel position reference and the PI controller steers twowheeled robot rotation around the vertical axis. A software in $M A T L A B^{\circledR} /$ Simulink environment intended for design and generation of control code which is embedded in a Texas Instruments Digital Signal Controller is developed. Simulation and experimental results of system performance are given that confirm the efficiency of the control system developed.

\section{Introduction}

Two-wheeled robots have several applications which make them useful both from theoretical and practical point of view [1]. The most popular commercial product, built on the idea of self- balancing two-wheeled robot is the Segway ${ }^{\circledR}$ Personal Transporter (PT), produced by Segway Inc., USA [2]. Another self-balancing two-wheeled robot NXTway-GS [3], built on the basis of the LEGO ${ }^{\circledR}$ Mindstorms NXT developer kit, is widely used in education. Also, the telepresence and video conferencing two-wheeled robot Double $^{\circledR}[4]$ is very popular recently. The two-wheeled robots have dynamics which is similar to the inverted pendulum dynamics so that they are inherently unstable and should be stabilized around the vertical position using a control system. Linear-quadratic or proportional-integralderivative (PID) control laws are usually implemented in order to achieve vertical stabilization and desired position in the horizontal plane [5].

In this paper we present the design and experimentation of a control system of two-wheeled robot which implements a cascade discrete-time Linear-Quadratic Regulator (LQR) with Kalman filter of $17^{\text {th }}$ order for stabilization in the vertical plane in presence of several noises. The reason to use such a controller for vertical stabilization is that it is tuned easier than the controllers of centralized (non-cascade) type. The cascade controllers also have several other features which make them attractive in the control systems design [6]. In respect to the robot rotation around the vertical axis a proportional-integral (PI) controller combined with a $2^{\text {nd }}$ order Kalman filter is used which ensures sufficient tracking accuracy. Due to the lack of accurate analytical robot model, the control system design is done by using a model built with the aid of an identification procedure. A software in MATLAB ${ }^{\circledR} /$ Simulink environment is developed for design and generation of control code which is embedded in the Texas Instruments Digital Signal Controller TMS320F28335. Results from the simulation of the closedloop system as well as experimental results obtained during the real-time implementation of the designed controller are given. The experimental results confirm the efficiency of the technical solution implemented.

\section{Control System Design}

\subsection{Experimental Set-Up}

A photo and kinematic diagram of the developed by the authors two-wheeled robot are shown in figures 1 and 2 , respectively.

The robot is constructed from 4 vertically connected plastic platforms. At the bottom platform are situated 2 DC motors, together with gearboxes, wheels and magnetic encoders on the shafts. Motors are controlled by power amplifier qik2s $12 v 10$ receiving commands from the processor. The next platform hosts the Spectrum Digital eZdspTMF28335 development board, which supports Texas Instruments DSP TMS320F28335. In the DSP a stabilizing controller for the robot and data acquisition (DAQ) system is embedded to allow closed-loop identification. Stabilization is achieved through inertial measurement unit ADIS16350 containing three orthogonal MEMS gyroscopes. This unit is mounted on the third platform. The robot is powered by 3 cell LiPo $12 \mathrm{~V}$ battery situated on the uppermost platform. The input signals $\left(\dot{u}_{1}, \dot{u}_{2}\right)$ to the motors are PWM duty cycles sent over RS232 link to the power amplifier forming PWM voltage waveforms for the motors. The output signals are wheels angular rates $\left(\dot{\theta}_{1}, \dot{\theta}_{2}\right)$, body tilt rate $(\dot{\varphi})$ and body yaw rate $(\dot{\psi})$. Real-time data acquisition system is organized around wireless communication channel based on Bluetooth module.

\subsection{Design Methodology}

The block-diagram of the two-wheeled robot control system is shown in figure 3. In respect to the stabilization in upper equilibrium state the robot is described by ARMAX and BJ models [7] obtained by means of identification procedures. The ARMAX model with structure parameters $n a=7, n b=7, n c=7, n k=3$ gives the link between the control signal $u$ and the rate $\dot{\varphi}$, while the BJ model with structure parameters $n b=3, n f=7, n c=3, n d=3, n k=1$ 


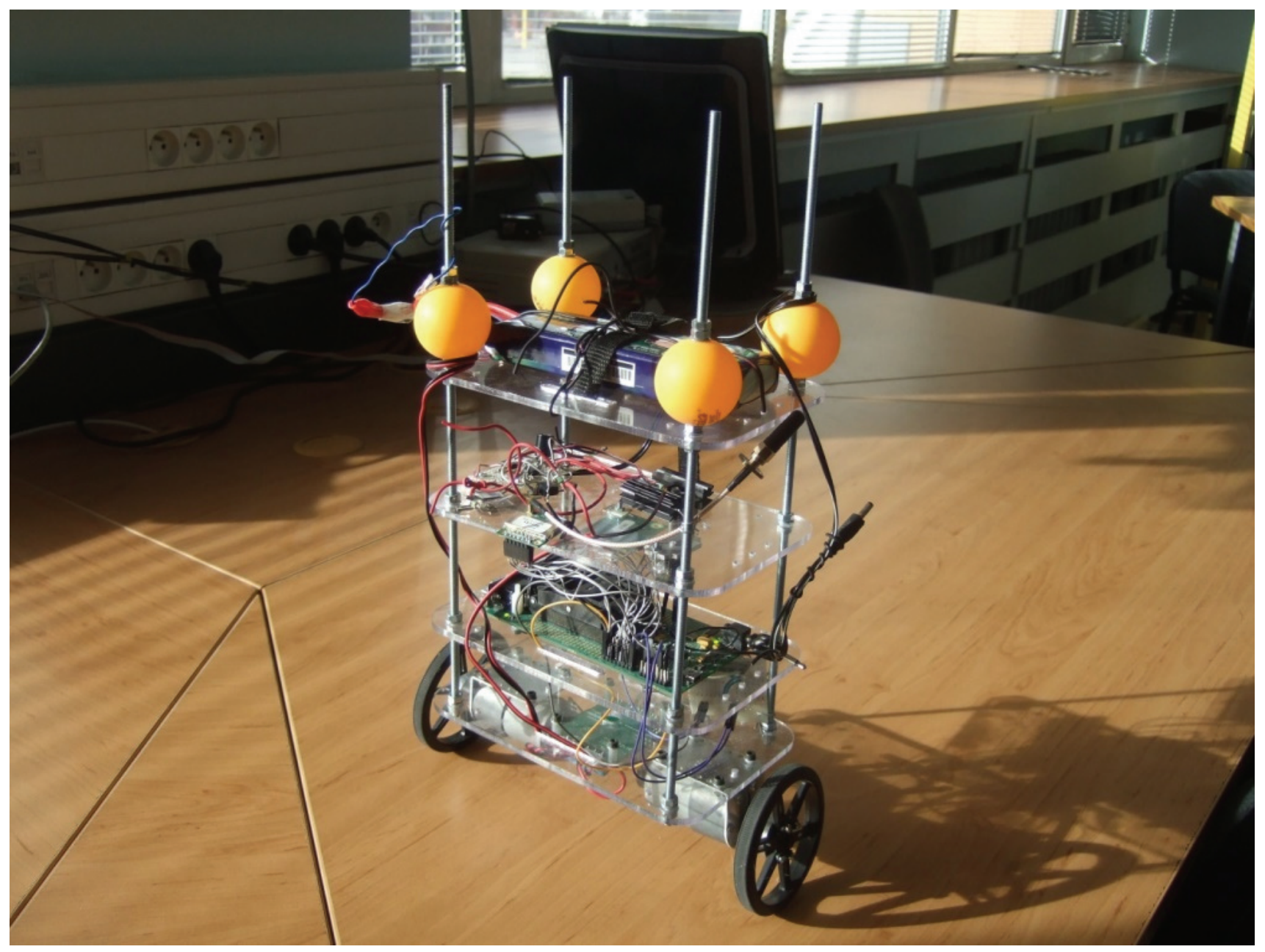

Figure 1. Two-wheeled robot in self-balancing mode
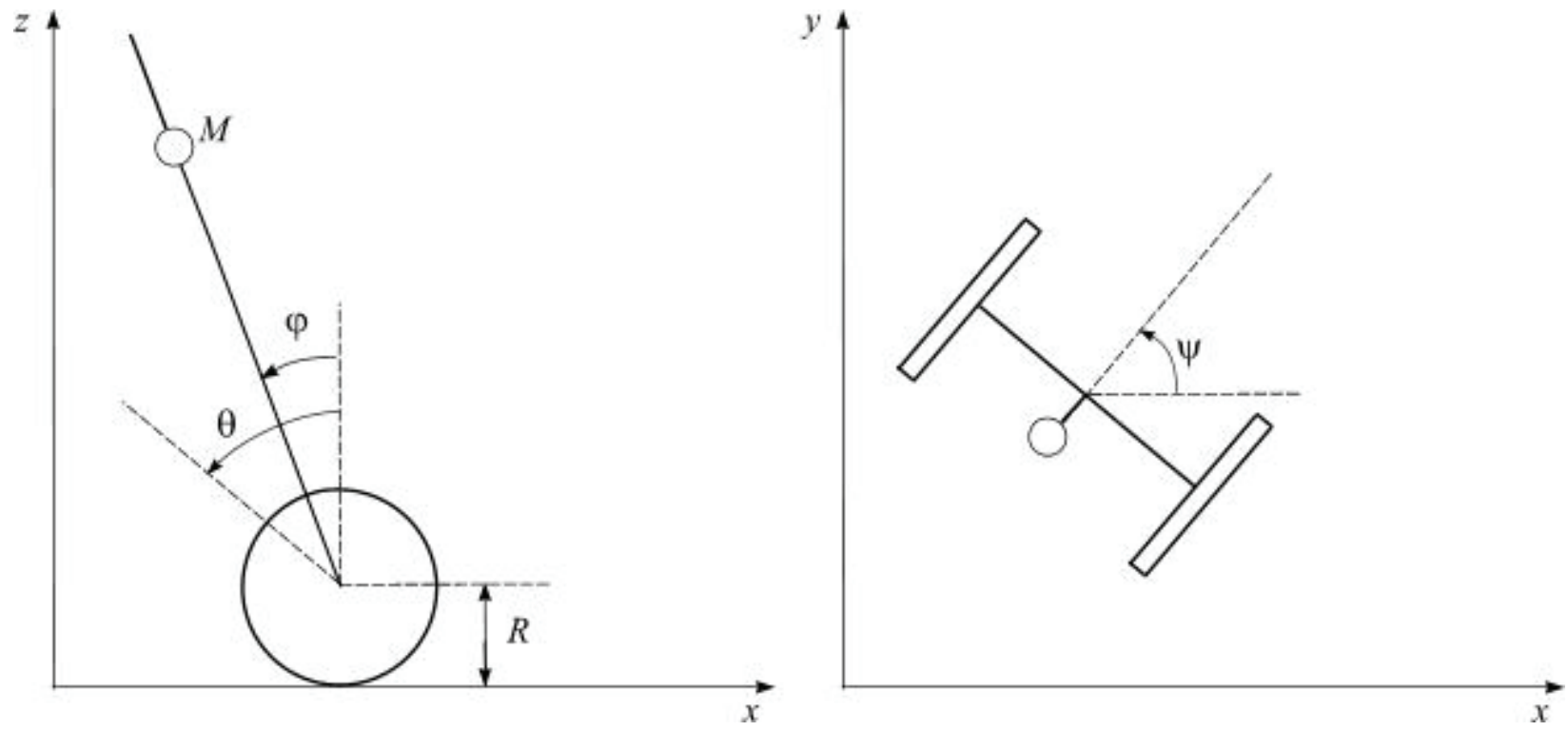

Figure 2. Robot motion in vertical and horizontal planes 


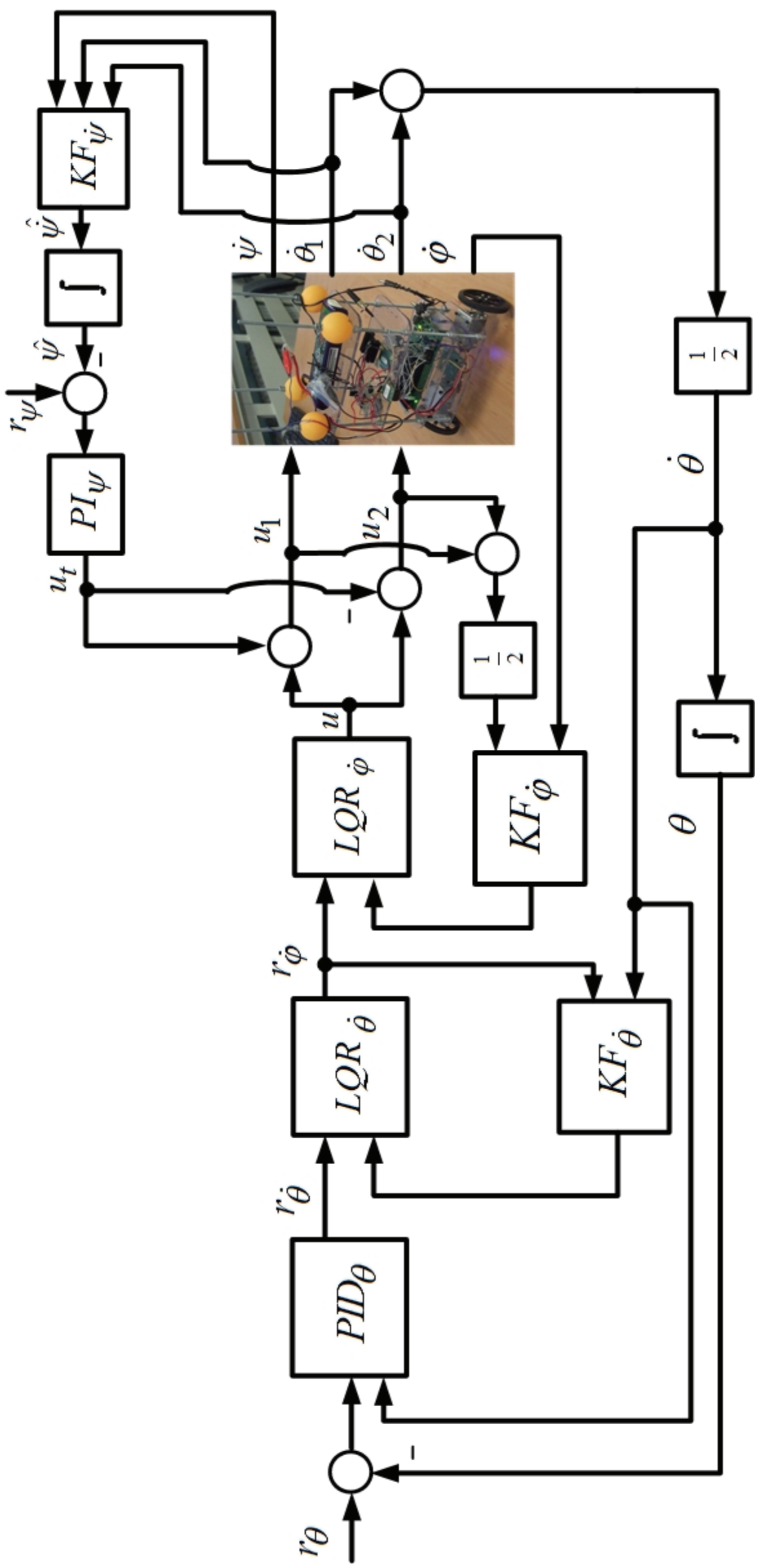

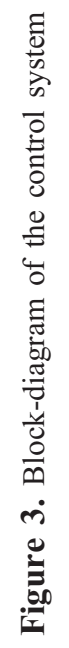


gives the link between the rates $\dot{\varphi}$ and $\dot{\theta}=\left(\dot{\theta}_{1}+\dot{\theta}_{2}\right) / 2$. These models are transformed into models with input multiplicative uncertainty which are used latter on in the closed-loop frequency domain analysis. In the design of cascade LQR controller, ARMAX and BJ models for nominal parameter values are transformed into the state space,

(1)

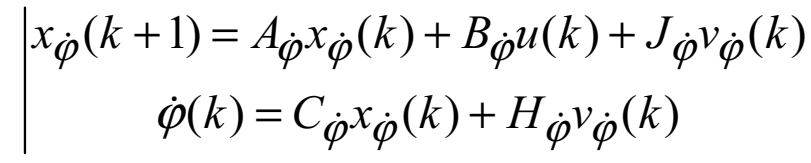

$$
\begin{aligned}
& \text { (2) } \\
& \mid \begin{array}{c}
x_{\dot{\theta}}(k+1)=A_{\dot{\theta}^{x}} \dot{\theta}(k)+B \dot{\theta} \dot{\varphi}(k)+J_{\dot{\theta}^{v}} \dot{\theta}^{(k)} \\
\dot{\theta}(k)=C_{\dot{\theta}^{x}} \dot{\theta}^{(k)+H_{\dot{\theta}}} \dot{\theta}_{\dot{\theta}}(k)
\end{array}
\end{aligned}
$$

where

$$
x_{\dot{\varphi}}(k)=\left[\begin{array}{llll}
x_{\dot{\varphi} 1}(k) & x_{\dot{\varphi} 2}(k) \quad \ldots \quad x_{\dot{\varphi} 9}(k)
\end{array}\right]^{T}, x_{\dot{\theta}}(k)=\left[\begin{array}{lll}
x_{\dot{\theta} 1}(k) & \ldots & x_{\dot{\theta} 6}(k)
\end{array}\right]^{T}
$$

are the corresponding state vectors, $v_{\dot{\varphi}}(k)$ and $v_{\dot{\theta}}(k)$ are discrete-time white Gaussian noises with variations $D v_{\dot{\varphi}}=\mathrm{D} v_{\dot{\theta}}=1$, and $A_{\dot{\varphi}}, B_{\dot{\varphi}} ; C_{\dot{\varphi}}, H_{\dot{\varphi}}, A_{\dot{\theta}} ; B_{\dot{\theta}} ; C_{\theta} ; H_{\theta} \cdot$ are matrices with corresponding dimensions containing the model parameters.

\section{Design of astatic $L Q R$ controller for $\dot{\varphi}\left(L Q R_{\dot{\varphi}}\right)$}

The controller $L Q R_{\dot{\varphi}}$ from the block-diagram in figure 3 under complete information about the state is described by

$$
\text { (3) } u(k)=-K_{\dot{\varphi}} x_{\dot{\varphi}}(k)+K_{\dot{\varphi}_{i}} x_{\dot{\varphi}_{i}}(k), x_{\dot{\varphi}_{i}}(k+1)=x_{\dot{\varphi}_{i}}(k)+T_{0}\left(r_{\dot{\varphi}}(k)-\dot{\varphi}(k)\right)
$$

where $K_{\dot{\varphi}}$ and $K_{\dot{\varphi}_{1}}$ are matrices with controller parameters, $r_{\dot{\varphi}}(k)$ is the reference of $\dot{\varphi}(k)$ and $T_{0}=0.005 \mathrm{~s}$ is the sampling interval. Introduce the extended state vector

$$
\text { (4) } \quad \bar{x}_{\dot{\varphi}}(k)=\left[\begin{array}{ll}
x_{\dot{\varphi}_{i}}(k) & x_{\dot{\varphi}}(k)
\end{array}\right]^{T} \text {. }
$$

From equations (1), (3) and (4), for the control system in respect to $\dot{\varphi}$ one obtains the following equation:

$$
\begin{aligned}
& \bar{x}_{\dot{\varphi}}(k+1)=\bar{A}_{\dot{\varphi}} \bar{x}_{\dot{\varphi}}(k)+\bar{B}_{\dot{\varphi}} u(k)+\bar{G}_{\dot{\varphi}^{r}} \dot{\varphi}(k)+\bar{J}_{\dot{\varphi}} v_{\dot{\varphi}}(k), u(k)=-\bar{K}_{\dot{\varphi}} \bar{x}_{\dot{\varphi}}, \\
& \dot{\varphi}(k)=\bar{C}_{\dot{\varphi}^{x}} \bar{x}_{\dot{\varphi}}(k)+H_{\dot{\varphi}} \dot{\varphi}_{\dot{\varphi}}(k),
\end{aligned}
$$

where

$$
\bar{A}_{\dot{\varphi}}=\left|\begin{array}{cc}
1 & -T_{0} C_{\dot{\varphi}} \\
0_{9 \times 1} & A_{\dot{\varphi}}
\end{array}\right|, \bar{B}_{\dot{\varphi}}=\left|\begin{array}{c}
0 \\
B_{\dot{\varphi}}
\end{array}\right|, \bar{J}_{\dot{\varphi}}=\left|\begin{array}{c}
-T_{0} H_{\dot{\varphi}} \\
J_{\dot{\varphi}}
\end{array}\right|, \bar{G}_{\dot{\varphi}}=\left|\begin{array}{c}
T_{0} r_{\dot{\varphi}} \\
0_{9 \times 1}
\end{array}\right|, \bar{C}_{\dot{\varphi}}=\left|\begin{array}{ll}
0 & C_{\dot{\varphi}}
\end{array}\right|, \bar{K}_{\dot{\varphi}}=\mid \begin{array}{cc}
-K_{\dot{\varphi}_{i}} & K_{\dot{\varphi}} \mid
\end{array}
$$

In the controller design we use the quadratic performance index

(6) $J_{\dot{\varphi}}=\sum_{k=1}^{\infty} \bar{x}_{\dot{\varphi}}^{T}(k) Q_{\dot{\varphi}} \bar{x}_{\dot{\varphi}}(k)+u^{T}(k) R_{\dot{\varphi}} u(k), Q_{\dot{\varphi}}=\left[\begin{array}{cc}4000 & 0 \\ 0 & 2 C_{\dot{\varphi}}^{T} C_{\dot{\varphi}}\end{array}\right], R_{\dot{\varphi}}=3$.

The controller matrix $\bar{K}_{\dot{\varphi}}$, which minimizes (6), is determined by

(7) $\bar{K}_{\dot{\varphi}}=\left(R_{\dot{\varphi}}+\bar{B}_{\dot{\varphi}}^{T} P_{\dot{\varphi}} \bar{B}_{\dot{\varphi}}\right) \bar{B}_{\dot{\varphi}}^{T} P_{\dot{\varphi}} \bar{A}_{\dot{\varphi}}$,

where the matrix $P_{\dot{\varphi}}$ is the positive definite solution of the equation

(8) $\bar{A}_{\dot{\varphi}}^{T} P_{\dot{\varphi}} \bar{A}_{\dot{\varphi}}-P_{\dot{\varphi}}-\bar{A}_{\dot{\varphi}}^{T} P_{\dot{\varphi}} \bar{B}_{\dot{\varphi}}\left(R_{\dot{\varphi}}+\bar{B}_{\dot{\varphi}}^{T} P_{\dot{\varphi}} \bar{B}_{\dot{\varphi}}\right)^{-1} \bar{B}_{\dot{\varphi}}^{T} P_{\dot{\varphi}} \bar{A}_{\dot{\varphi}}+Q_{\dot{\varphi}}=0$

The control $u(k)$ of the system shown in figure 3 is determined by the expression 
(9)

$$
u(k)=K_{\dot{\varphi}_{i}} x_{\dot{\varphi}_{i}}(k)-K_{\dot{\varphi}} \hat{x}_{\dot{\varphi}}(k), x_{\dot{\varphi}_{i}}(k+1)=x_{\dot{\varphi}_{i}}(k)+T_{0}\left(r_{\dot{\varphi}}(k)-C_{\dot{\varphi}} \hat{x}_{\dot{\varphi}}(k)\right),
$$

where $\hat{x}_{\dot{\varphi}}(k)$ is an estimate of the state vector $x_{\dot{\varphi}}(k)$, obtained by a Kalman filter in respect to $\dot{\varphi}\left(K F_{\dot{\varphi}}\right)$

$$
\hat{x}_{\dot{\varphi}}(k+1)=A_{\dot{\varphi}} \hat{x}_{\dot{\varphi}}(k)+B_{\dot{\varphi}} u(k)+K_{K F_{\dot{\varphi}}}\left(\dot{\varphi}(k+1)-C_{\dot{\varphi}} B_{\dot{\varphi}} u(k)-C_{\dot{\varphi}} A_{\dot{\varphi}} \hat{x}_{\dot{\varphi}}(k)\right)
$$

The coefficient $K_{K F_{\dot{\varphi}}}$ of $K F_{\dot{\varphi}}$ is determined by the expression

$$
K_{K F_{\dot{\varphi}}}=D C_{\dot{\varphi}}^{T}\left(C_{\dot{\varphi}} D C_{\dot{\varphi}}^{T}+0.00001\right)^{-1}, D=F_{\dot{\varphi}} D_{e} F_{\dot{\varphi}}^{T}+J_{\dot{\varphi}} D_{v_{\dot{\varphi}}} J_{\dot{\varphi}}^{T}
$$

where $D_{e}$ is the positive definite solution of the equation

$$
D_{e}=D-D C_{\dot{\varphi}}^{T}\left(C_{\dot{\varphi}} D C_{\dot{\varphi}}^{T}+0.00001\right)^{-1} C_{\dot{\varphi}} D
$$

From equations (1), (4), (9) and (10) one obtains for the description of the control system under incomplete information in respect to $\dot{\varphi}$ the following equation:

$$
\begin{aligned}
& \mid \begin{array}{l}
\tilde{x}_{\dot{\varphi}}(k+1)=\tilde{A}_{\dot{\varphi}} \tilde{x}_{\dot{\varphi}}(k)+\tilde{G}_{\dot{\varphi}} \dot{\varphi}(k)+\tilde{J}_{\dot{\varphi}^{v}} \dot{\varphi}(k), \\
\dot{\varphi}(k)=\tilde{C}_{\dot{\varphi}} \tilde{x}_{\dot{\varphi}}(k)+H_{\dot{\varphi}} \dot{\varphi}^{(k),}
\end{array} \\
& \tilde{A}_{\dot{\varphi}}=\left|\begin{array}{ccc}
1 & 0_{1 \times 9} & -T_{0} C_{\dot{\varphi}} \\
B_{\dot{\varphi}} K_{\dot{\varphi}_{i}} & A_{\dot{\varphi}} & -B_{\dot{\varphi}} K_{\dot{\varphi}} \\
B_{\dot{\varphi}} K_{\dot{\varphi}_{i}}-K_{K F_{\dot{\varphi}}} C_{\dot{\varphi}} B_{\dot{\varphi}} K_{\dot{\varphi}_{i}} & 0_{9 \times 9} & A_{\dot{\varphi}}-K_{K F_{\dot{\varphi}}} C_{\dot{\varphi}} A_{\dot{\varphi}}-B_{\dot{\varphi}} K_{\dot{\varphi}}+K_{K F_{\dot{\varphi}}} C_{\dot{\varphi}} B_{\dot{\varphi}} K_{\dot{\varphi}}
\end{array}\right|,
\end{aligned}
$$

$$
\tilde{G}_{\dot{\varphi}}=\left|\begin{array}{c}
T_{0} \\
0_{9 \times 1} \\
0_{9 \times 1}
\end{array}\right|, \tilde{J}_{\dot{\varphi}}=\left|\begin{array}{c}
0 \\
J_{\dot{\varphi}} \\
0_{9 \times 1}
\end{array}\right|, \tilde{C}_{\dot{\varphi}}=\left|\begin{array}{lll}
0 & C_{\dot{\varphi}} & 0_{1 \times 9}
\end{array}\right| \text {. }
$$

\section{Design of astatic $L Q R$ controller for $\dot{\theta}\left(L Q R_{\theta}\right)$}

To simplify the design, the system (13) in respect to measurable signals is approximated by the second-order system

$$
\mid \begin{aligned}
& x_{\dot{\varphi}_{2}}(k+1)=A_{\dot{\varphi}_{2}} x_{\dot{\varphi}_{2}}(k)+B_{\dot{\varphi}_{2}} r_{\dot{\varphi}}(k) \\
& \dot{\varphi}(k)=C_{\dot{\varphi}_{2}} x_{\dot{\varphi}_{2}}(k)
\end{aligned} .
$$
obtains

The system (14) is connected in serial to the model (2), so that for the plant in respect to the controller $L Q R_{q}$ one

$$
\mid \begin{aligned}
& \bar{x}_{\dot{\theta}}(k+1)=\bar{A}_{\dot{\theta}} \bar{x}_{\dot{\theta}}(k)+\bar{B}_{\dot{\theta}} r_{\dot{\varphi}}(k)+\bar{J}_{\dot{\theta}} \dot{\theta}_{\dot{\theta}}(k) \\
& \dot{\theta}(k)=\bar{C}_{\dot{\theta}} \bar{x}_{\dot{\theta}}(k)+H_{\dot{\theta}} v_{\dot{\theta}}(k)
\end{aligned},
$$

where

$$
\bar{A}_{\dot{\theta}}=\left[\begin{array}{cc}
A_{\dot{\varphi}_{2}} & 0_{2 \times 6} \\
B_{\dot{\theta}} C_{\dot{\varphi}_{2}} & A_{\dot{\theta}}
\end{array}\right], \bar{B}_{\dot{\theta}}=\left|\begin{array}{c}
B_{\dot{\varphi}_{2}} \\
0_{6 \times 1}
\end{array}\right|, \bar{J}_{\dot{\theta}}=\left|\begin{array}{c}
0_{2 \times 1} \\
J_{\dot{\theta}}
\end{array}\right|, \bar{C}_{\dot{\theta}}=\left|\begin{array}{ll}
0_{1 \times 2} & C_{\dot{\theta}}
\end{array}\right| .
$$


The output $r_{\dot{\varphi}}(k)_{\text {of the controller }} L Q R_{\dot{\theta}}$ is determined by the expression

(16) $r_{\dot{\varphi}}(k)=K_{\dot{\theta}_{i}} \bar{x}_{\dot{\theta}_{i}}(k)-K_{\dot{\theta}} \hat{\bar{x}}_{\dot{\theta}}(k), \bar{x}_{\dot{\theta}_{i}}(k+1)=\bar{x}_{\dot{\theta}_{i}}(k)+T_{0}\left(r_{\dot{\theta}}(k)-\bar{C}_{\dot{\theta}} \hat{\bar{x}}_{\dot{\theta}}(k)\right)$,

where $\hat{\bar{x}}_{\dot{\theta}}(k)$ is an estimate of the state vector $\bar{x}_{\dot{\theta}}(k)$, which is obtained by the Kalman filter in respect to $\dot{\theta}$ ( $K F_{\dot{\theta}}$ ), $K_{\dot{\theta}}$ and $K_{\dot{\theta}}$ are controller parameters. The equations of $K F_{\dot{\theta}}$ are analogous to these of $K F_{\dot{\varphi}}$ written in respect to (15). Similarly to (13), for the description of control system one obtains

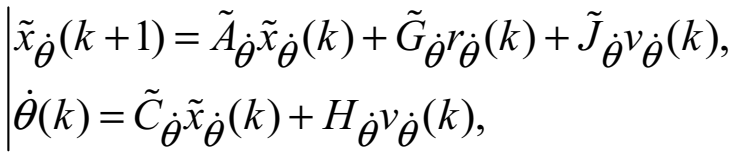

$$
\begin{aligned}
& \tilde{A}_{\dot{\theta}}=\left|\begin{array}{ccc}
1 & 0_{1 \times 8} & -T_{0} \bar{C}_{\dot{\theta}} \\
\bar{B}_{\dot{\theta}} K_{\dot{\theta}_{i}} & \bar{A}_{\dot{\theta}} & -\bar{B}_{\dot{\theta}} K_{\dot{\theta}} \\
\bar{B}_{\dot{\theta}} K_{\dot{\theta}_{i}}-K_{K F_{\dot{\theta}}} \bar{C}_{\dot{\theta}} \bar{B}_{\dot{\theta}} K_{\dot{\theta}_{i}} & 0_{8 \times 8} & \bar{A}_{\dot{\theta}}-K_{K F_{\dot{\theta}}} \bar{C}_{\dot{\theta}} \bar{A}_{\dot{\theta}}-\bar{B}_{\dot{\theta}} K_{\dot{\theta}}+K_{K F_{\dot{\theta}}} \bar{C}_{\dot{\theta}} \bar{B}_{\dot{\theta}} K_{\dot{\theta}}
\end{array}\right| \text {, } \\
& \tilde{G}_{\dot{\theta}}=\left|\begin{array}{c}
T_{0} \\
0_{8 \times 1} \\
0_{8 \times 1}
\end{array}\right|, \tilde{J}_{\dot{\theta}}=\left|\begin{array}{c}
0 \\
\bar{J}_{\dot{\theta}} \\
0_{8 \times 1}
\end{array}\right|, \tilde{C}_{\dot{\theta}}=\left|\begin{array}{lll}
0 & C_{\dot{\theta}} & 0_{1 \times 8}
\end{array}\right|, \tilde{x}_{\dot{\theta}}(k)=\left|\begin{array}{c}
\bar{x}_{\dot{\theta}_{i}}(k) \\
\bar{x}_{\dot{\theta}}(k) \\
\hat{\bar{x}}_{\dot{\theta}}(k)
\end{array}\right|
\end{aligned}
$$

where $K_{K F_{\dot{\theta}}}$ is the gain of $K F_{\dot{\theta}}$. The design of the controller $L Q R_{\dot{\theta}}$ is done for the quadratic performance index

$$
\begin{aligned}
& J_{\dot{\theta}}=\sum_{k=1}^{\infty}\left|\bar{x}_{\dot{\theta}_{i}}(k) \quad \bar{x}_{\dot{\theta}}(k)\right| Q_{\dot{\theta}}\left|\bar{x}_{\dot{\theta}_{i}}(k) \quad \bar{x}_{\dot{\theta}}(k)\right|^{T}+r_{\dot{\varphi}}^{T}(k) R_{\dot{\theta}} \dot{\varphi}_{\dot{\varphi}}(k), \\
& Q_{\dot{\theta}}=\left[\begin{array}{cc}
30 & 0 \\
0 & 10 \bar{C}_{\dot{\theta}}^{T} \bar{C}_{\dot{\theta}}
\end{array}\right], R_{\dot{\theta}}=30
\end{aligned}
$$

The controller parameters are determined according to equations (7) and (8) written in respect to

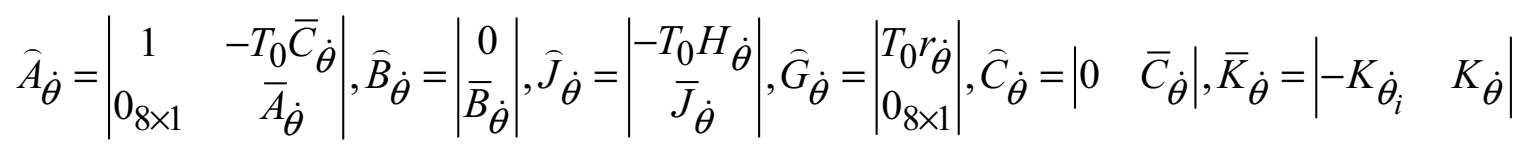

\section{Design of digital PID regulator for control of $\theta(P I D \theta)$}

After that the robot is stabilized in upper equilibrium state with the controllers $L Q R_{\varphi}$ and $L Q R_{\theta}$, it is necessary to tune the parameters of the controller $P I D_{\theta}$ which manipulates the angle $\theta$ of the wheels rotation. The control $r_{\theta}$ is determined by

$$
r_{\dot{\theta}}(k)=K_{\theta_{n}}\left(r_{\theta}(k)-\theta(k)\right)+K_{\theta_{i}} I_{\theta}(k)-K_{\theta_{d}} \dot{\theta}(k), I_{\theta}(k+1)=I_{\theta}(k)+T_{0}\left(r_{\theta}(k)-\theta(k)\right),
$$

where $r_{\theta}(k)$ is the reference of angle $\theta, K_{\theta_{n}}, K_{\theta_{i}}$ and $K_{\theta_{d}}$ are coefficients of proportionality, integration and differentiation. Based on the requirements to the frequency responses of the forward-backward robot motion control system and to the frequency responses to the controller $P I D_{\theta}$, the controller coefficients are chosen as $K_{\theta_{n}}=1.5, K_{\theta_{i}}=0.05$ and $K_{\theta_{d}}=0.07$. 


\section{Frequency domain analysis of the forward-backward} motion control system

Based on the plant model with input multiplicative uncertainty and on the designed controllers, a frequency domain analysis of the closed-loop system is done. The singular values of the closed-loop system, output sensitivity function and controller singular values used in the analysis are shown in figures 4-6, respectively.

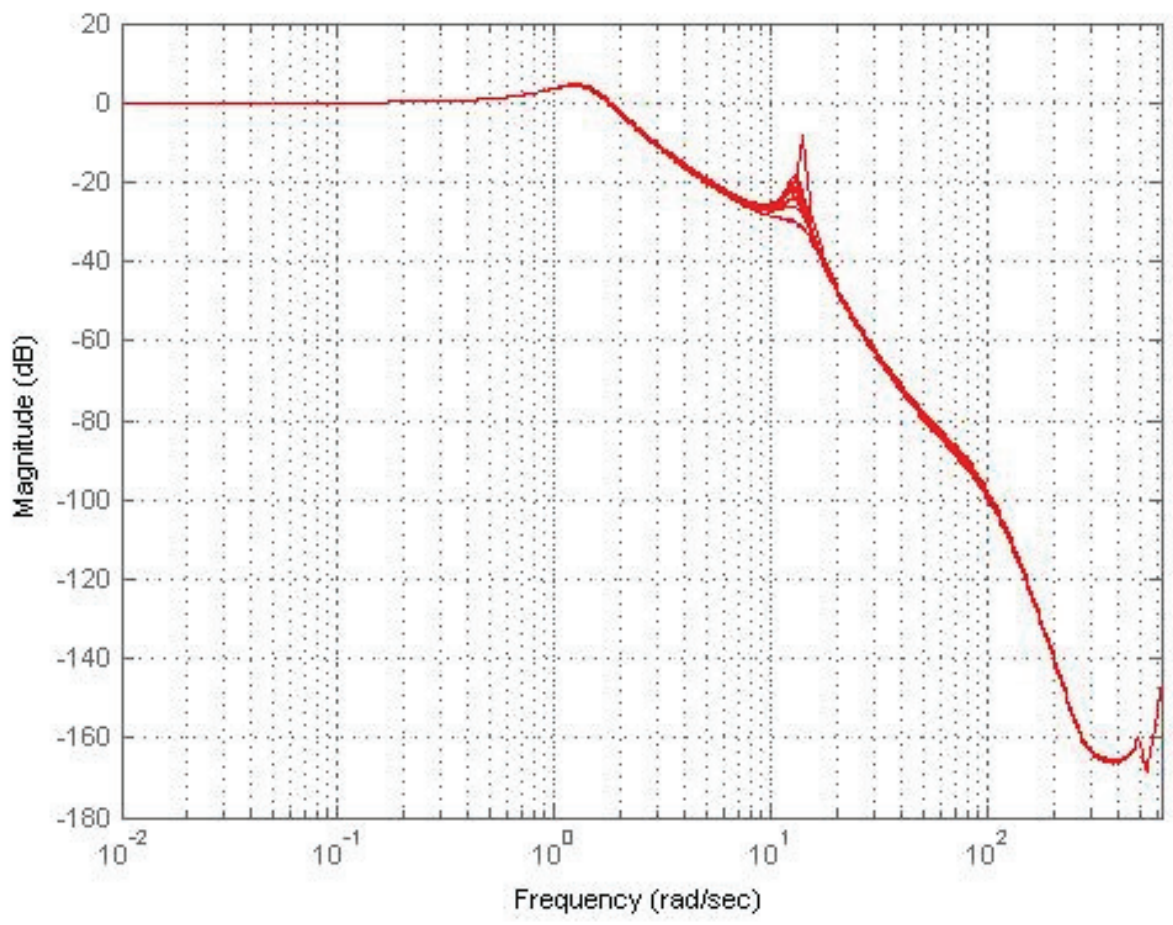

Figure 4. Closed-loop system singular value

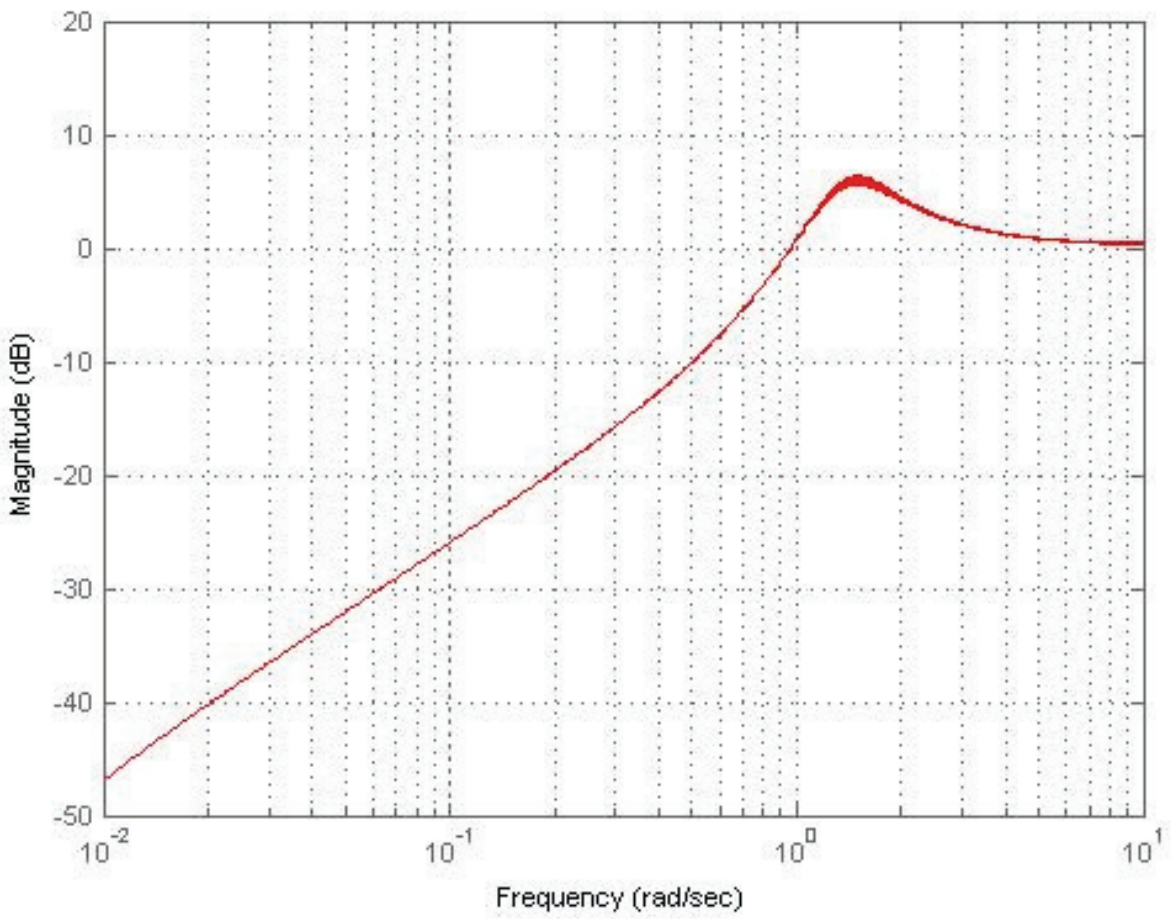

Figure 5. Output sensitivity function 


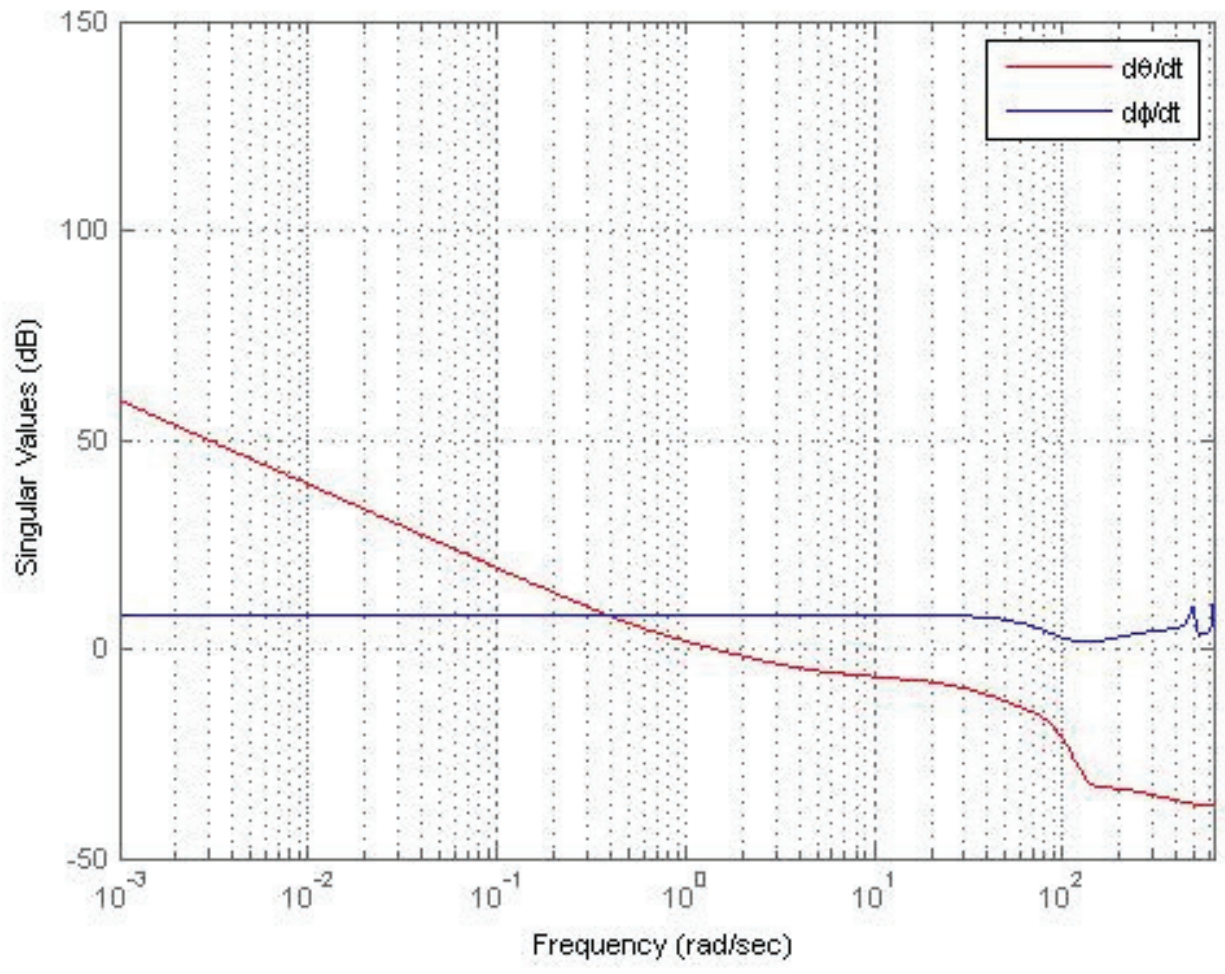

Figure 6. Controller singular values

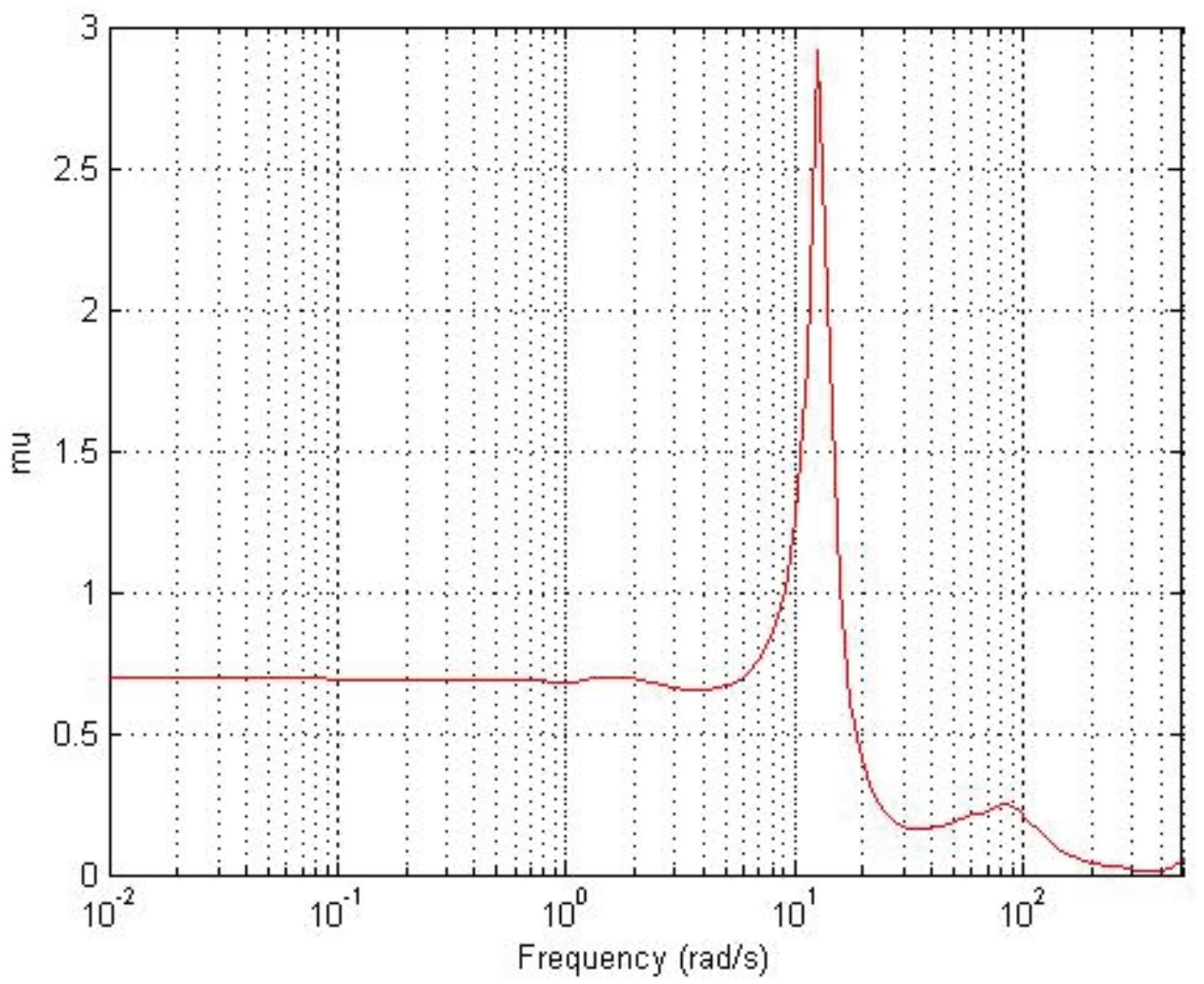

Figure 7. Robust stability 


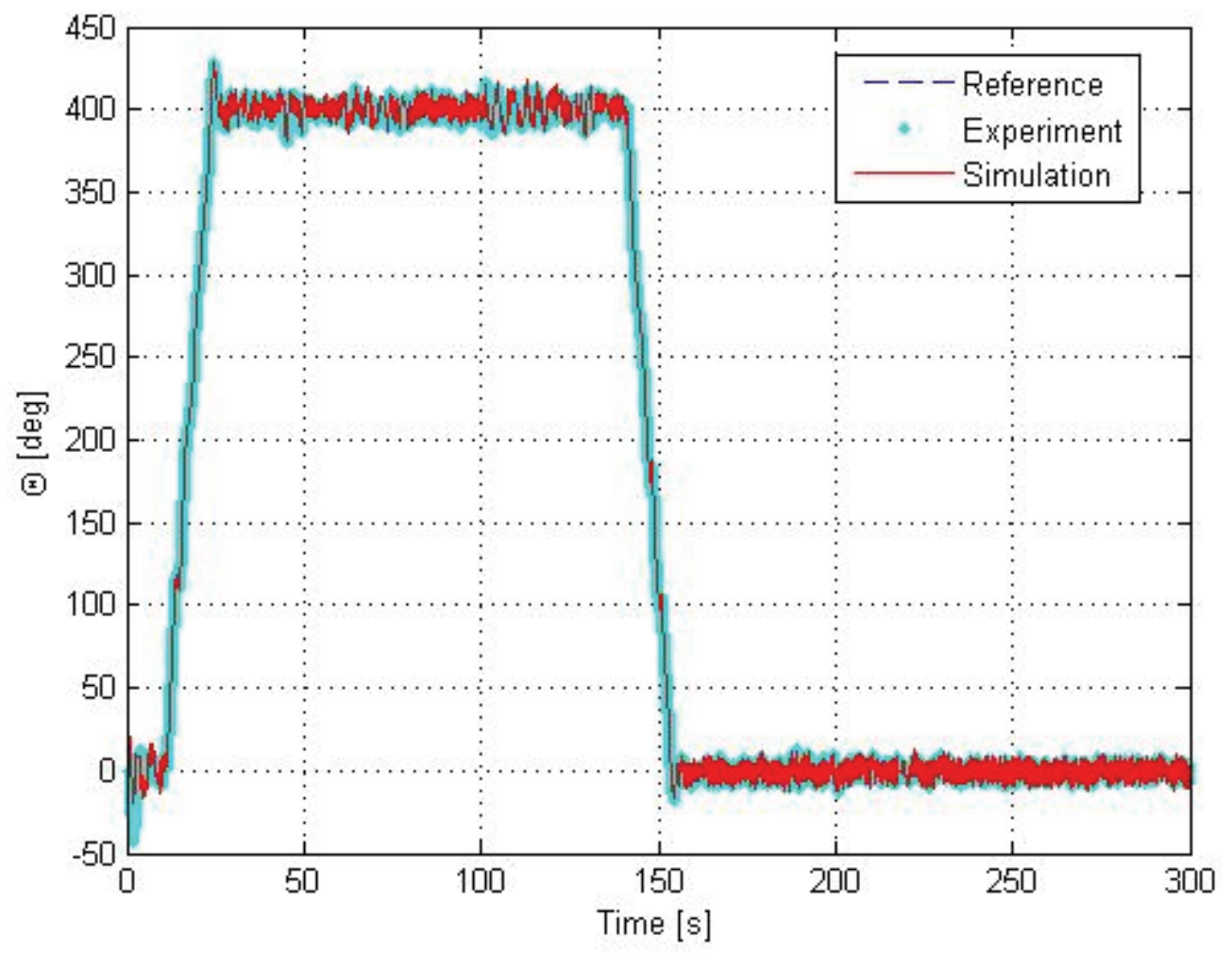

Figure 8. Position of robot wheels

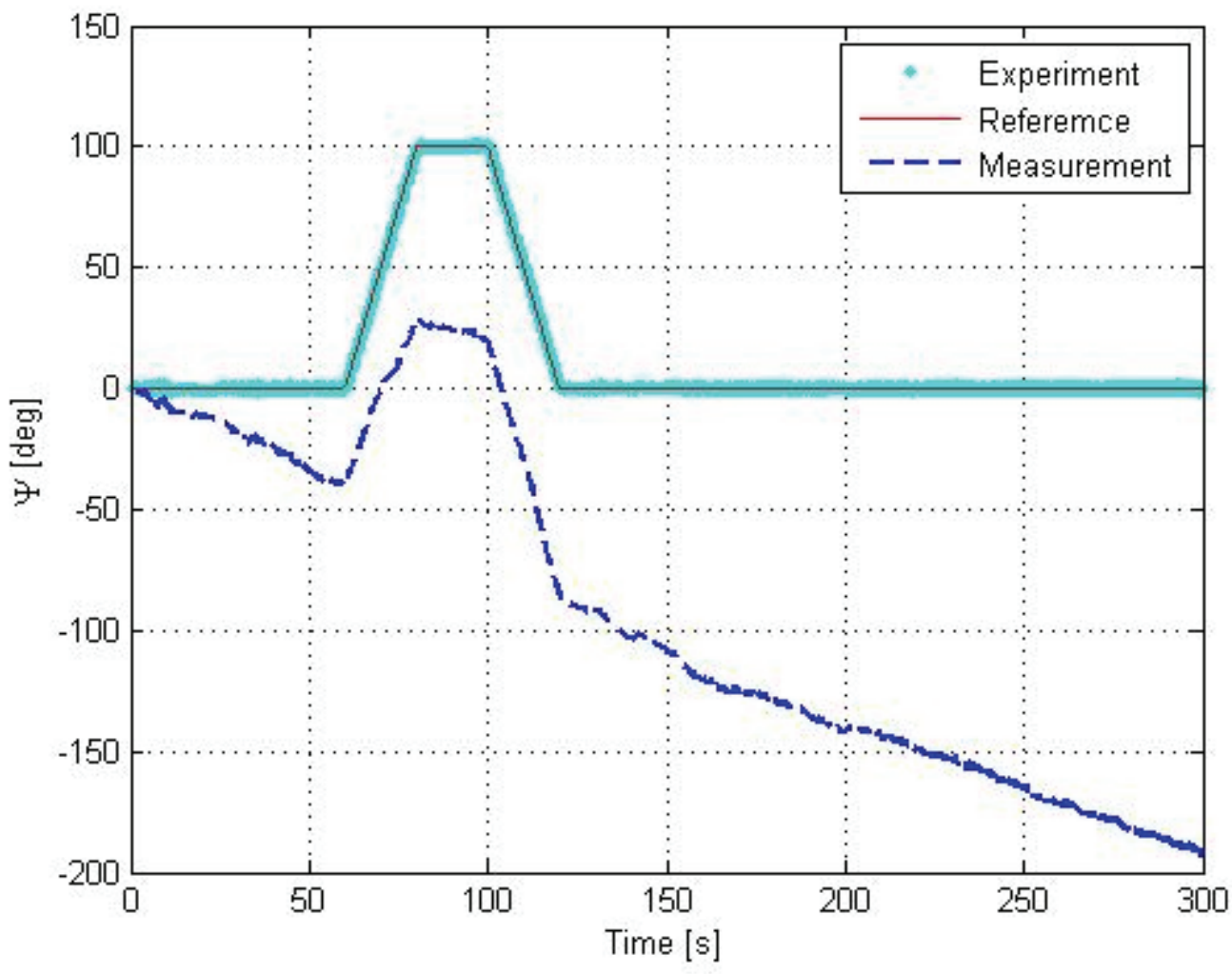

Figure 9. Angle of rotation around the vertical axis 


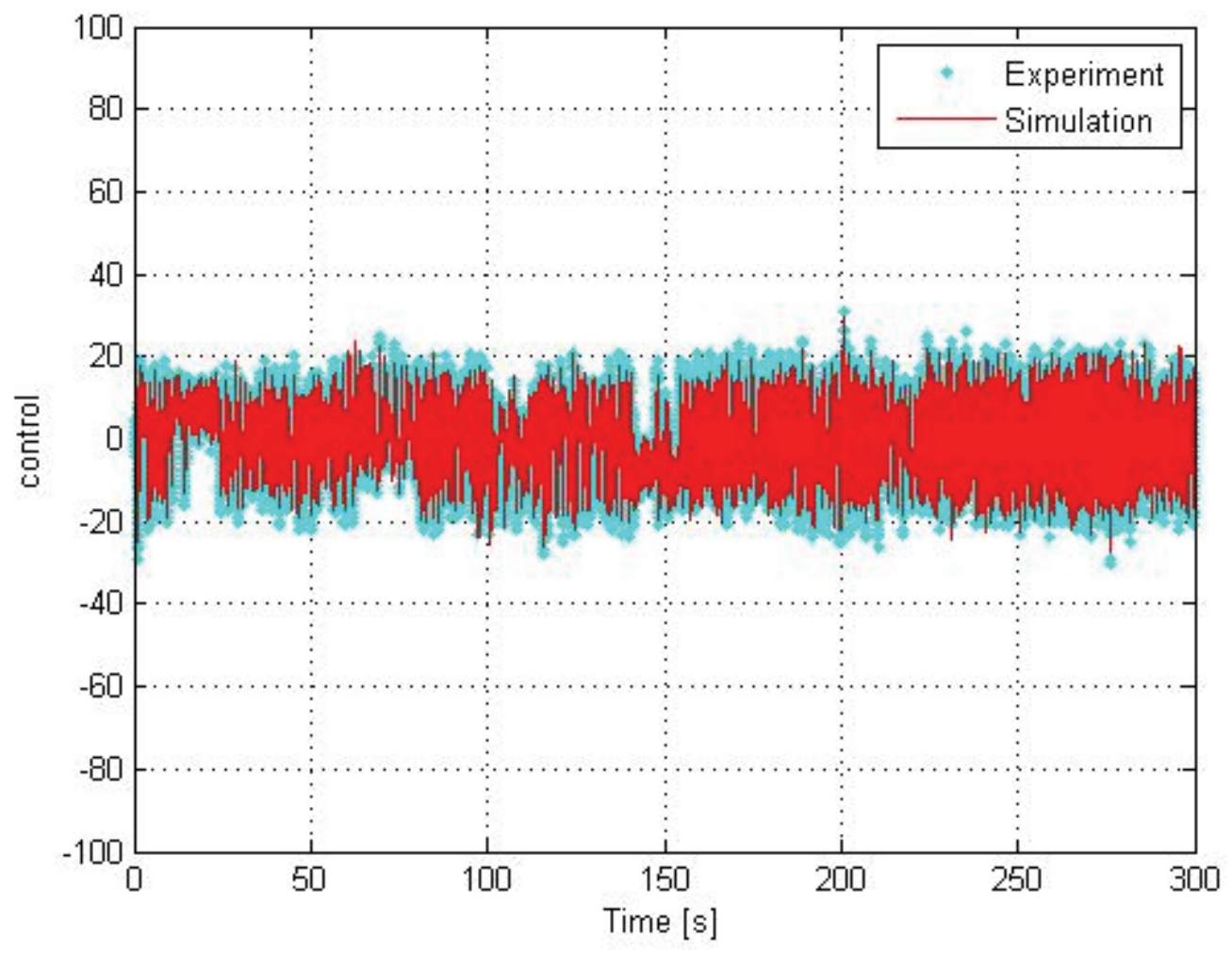

Figure 10. Control signal

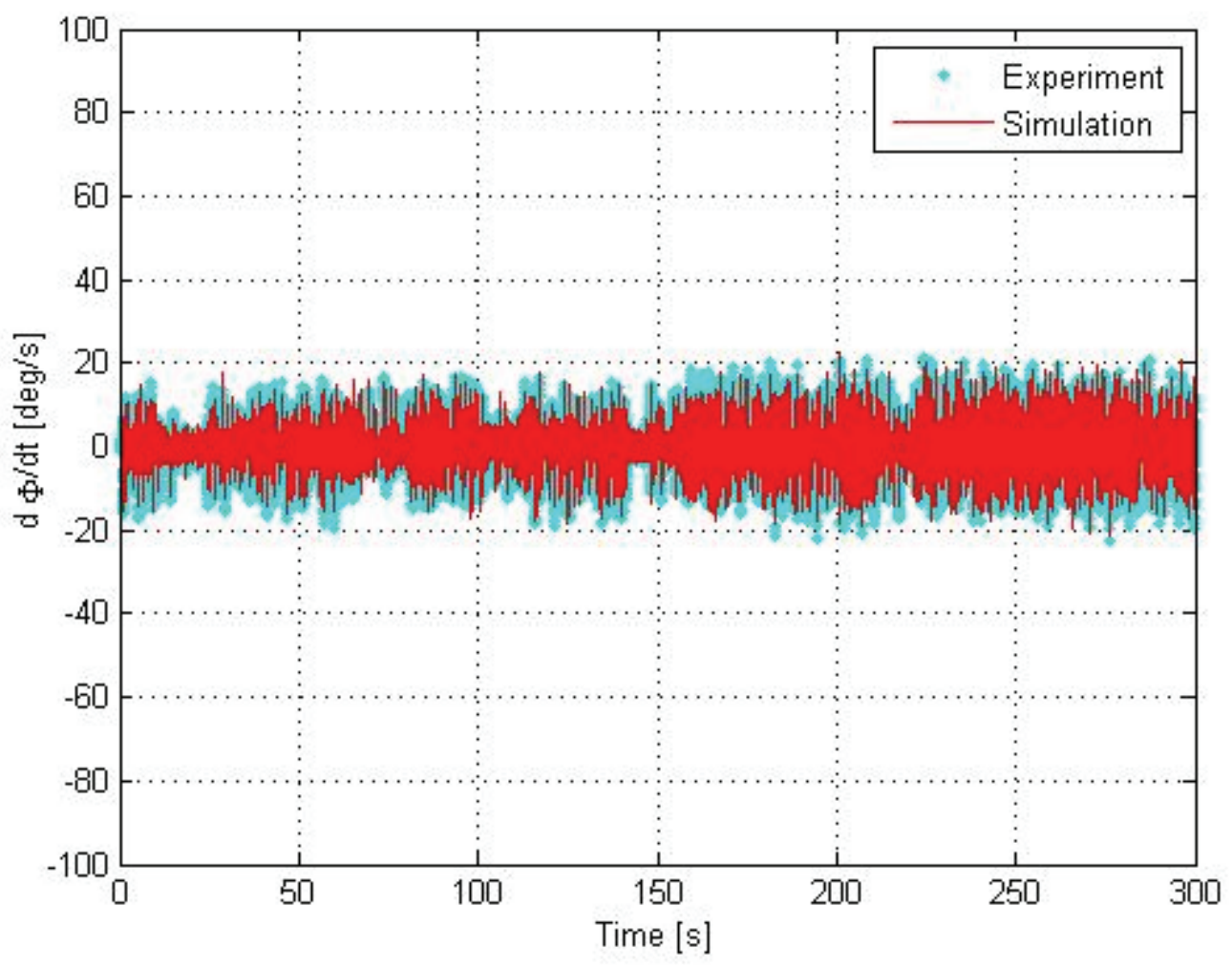

Figure 11. Tilt velocity of the robot body 
Clearly, the control system tracks without static errors the references of wheels position with frequency up to 1 $\mathrm{rad} / \mathrm{s}$ and the low frequency disturbances are sufficiently suppressed.

In figure 7 we represent the structured singular value ( $\mu$ ) [9] of the closed-loop uncertain system corresponding to the robust stability analysis.

The analysis of the robust stability shows that there is sufficient stability margin in the low frequency range while there are allowable uncertainties in the frequency range of $0.9 \div 19 \mathrm{rad} / \mathrm{s}$, that can make the system unstable. This is well known disadvantage of the linear quadratic regulators which make them applicable to systems with relatively small uncertainties.

\section{Digital PI controller for steering the robot motion around vertical axis}

In respect to the motion around vertical axis the robot is described by the determined through identification transfer functions

$$
\begin{aligned}
W_{\dot{\theta}_{1} u_{1}} & =\frac{8.5867}{0.1328 p+1}, W_{\dot{\theta}_{2} u_{2}}=\frac{8.7270}{0.1186 p+1}, \\
W_{\dot{\psi} \dot{\theta}_{1}} & =\frac{-0.2004}{0.0119 p+1}, W_{\dot{\psi} \dot{\theta}_{2}}=\frac{0.2009}{0.0119 p+1}
\end{aligned}
$$

where $u_{1}$ and $u_{2}$ are the control signals to the left and right wheel, respectively. The control signal of the PI controller

$(P I \psi)$ is determined by

$$
\text { (20) } u_{t}(k)=K_{\psi_{n}}\left(r_{\psi}(k)-\hat{\psi}(k)\right)+K_{\psi_{i}} I_{\psi}(k), I_{\psi}(k+1)=I_{\psi}(k)+T_{0}\left(r_{\psi}(k)-\hat{\psi}(k)\right) \text {, }
$$

where $r_{\psi}(k)$ is the reference of angle $\psi(k), K_{\psi_{n}}$ and $K_{\psi_{i}}$ are coefficients of proportionality and integration. In order to find a compromise between the performance of tracking $r_{\psi}(k)$ and the magnitude of the control signal $u_{t}(k)$, which is add to the output of forward-backward controller, it is chosen that $K_{\psi_{n}}=2$ and $K_{\psi_{i}}=0.01$. In expression (20) $\hat{\psi}(k)$ is an estimate of the angle $\psi(k)$, which is obtained as

$$
\text { (21) } \hat{\psi}(k+1)=\hat{\psi}(k)+T_{0} \hat{\dot{\psi}}(k) \text {, }
$$

where $\hat{\dot{\psi}}(k)$ is an estimate of the measured by gyro velocity $\dot{\psi}(k)$. In the computation of control one uses the es timate $\hat{\dot{\psi}}(k)$, taking into account that the measured by gyro velocity $\psi(k)$ is given by

$$
\text { (22) } \dot{\psi}(k)=\dot{\psi}_{m}(k)+\dot{\psi}_{b}(k),
$$

where $\psi_{b}(k)$ is the bias and $\psi_{m}(k)$ is the exact rotation rate around the vertical axis. The bias in (22) is determined from [8]

$$
\text { (23) } \quad \dot{\psi}_{b}(k+1)=\dot{\psi}_{b}(k)+b T_{0} v_{\dot{\psi}}(k),
$$

where $v_{\dot{\psi}}$ is a discrete-time white Gaussian noise with variation $D v_{\dot{\psi}}=1$. The estimate $\hat{\dot{\psi}}(k)$ is determined by PI Kalman filter $\left(K F_{\dot{\psi}}\right)$. Tis filter is designed in respect to an extended model which is obtained after discretization of the transfer functions (19) and taking into account the equations (22) and (23),

$$
\begin{aligned}
& \begin{array}{l}
\left|\begin{array}{l}
\dot{\psi}_{b}(k+1) \\
\dot{\psi}_{m}(k+1)
\end{array}\right|=\left|\begin{array}{cc}
1 & 0 \\
0 & A_{\dot{\psi}}
\end{array}\right| x_{\dot{\psi}}(k)+\left|\begin{array}{c}
0_{1 \times 2} \\
B_{\dot{\psi}}
\end{array}\right| \dot{\dot{\theta}}(k)+\left|\begin{array}{ccc}
b T_{0} & 0 & 0 \\
0 & 1 & 1
\end{array}\right| \bar{\eta}_{\dot{\psi}}(k), \\
\dot{\psi}(k)=\dot{\psi}_{m}(k)+\dot{\psi}_{b}(k),
\end{array} \\
& \overline{\dot{\theta}}(k)=\left|\dot{\theta}_{1}(k) \quad \dot{\theta}_{2}(k)\right|^{T}, \bar{\eta}_{\dot{\psi}}(k)=\left|\begin{array}{lll}
v_{\dot{\psi}}(k) & \eta_{\dot{\theta}_{1}}(k) & \eta_{\dot{\theta}_{2}}(k)
\end{array}\right|^{T},
\end{aligned}
$$

where $\eta_{\dot{\theta}_{1}}(k)$ and $\eta_{\dot{\theta}_{2}}(k)$ are uncorrelated white Gaussian ties with variations $D_{\dot{\theta}_{1}}=D_{\dot{\theta}_{2}}=9$. For $K F_{\dot{\psi}}$ one obtains noises representing the errors in measuring wheels veloci-

$$
\begin{aligned}
& \hat{\dot{\psi}}_{b}(k+1)=\hat{\dot{\psi}}_{b}(k)+K_{K F_{\dot{\psi}} i}\left(\dot{\psi}(k+1)-\hat{\dot{\psi}}_{b}(k)-A_{\dot{\psi}} \hat{\dot{\psi}}_{m}(k)-B_{\dot{\psi}} \overline{\dot{\theta}}(k)\right) \\
& \hat{\dot{\psi}}_{m}(k+1)=A_{\dot{\psi}} \hat{\dot{\psi}}_{m}(k)+B_{\dot{\psi}} \dot{\dot{\theta}}(k)+K_{K F_{\dot{\psi}}}\left(\dot{\psi}(k+1)-\hat{\dot{\psi}}_{b}(k)-A_{\dot{\psi}} \hat{\dot{\psi}}_{m}(k)-B_{\dot{\psi}} \overline{\dot{\theta}}(k)\right)
\end{aligned}
$$


where $K_{K F_{\dot{\psi}}}=\left|K_{K F_{\dot{\psi} i}} \quad K_{K F_{\dot{\psi} n}}\right|^{T}$ is the filter gain. It is determined according to expressions (11) and (12) written in respect to the model (24). The estimate $\hat{\dot{\psi}}(k)$ is determined from

(26) $\hat{\dot{\psi}}(k)=\dot{\psi}(k)-\hat{\dot{\psi}}_{b}(k)$.

\section{Experimental Results}

A simulation scheme of the control system and a specialized software in MATLAB ${ }^{\circledR} /$ Simulink environment is developed to implement the control code. With the aid of Simulink Coder and Code Composer Studio, a code is generated from this software which is embedded in the Texas Instruments Digital Signal Controller TMS320F28335 [10]. Several experiments are performed with the designed controllers and comparison is done with the simulation results. The results from experiment and simulation concerning the basic signals of the control system are shown in figures 8-11.

It is seen from figure 8 the perfect tracking of the wheels position reference and the independence of the forward-backward motion on the robot rotation around the vertical axis. The usage of $\hat{\psi}(k)$ instead of $\psi(k)$ ensures exact robot rotation around the vertical axis while a significant drift in the measured value of $\psi(k)$ is observed with the time, which is due to the integration of the gyro bias (figure 9). The system control signal has an acceptable magnitude and is significantly smaller than the magnitude constraints of \pm 50 (figure 10). The robot body tilt velocity is shown in figure 11.

\section{Conclusions}

The paper presents the developed cascade LQR controller, Kalman filters, PID and PI controllers intended to control the plane motion of a two-wheeled robot. The developed algorithms are embedded in the Digital Signal Processor TMS320F28335. Experimental and simulation results of the robot motion are given which demonstrate the performance of the embedded control system. The closeness between the experimental and simulation results shows the efficiency of the developed control system. Better results in respect to the robustness of the closed-loop system may be achieved by using $\mathrm{H}_{\infty}$ or $\mu$-controllers.

\section{References}

1. Campion, G., W. Chung. Wheeled Robots. In B. Siciliano and O. Khatib, Editors, Springer Handbook of Robotics, Chapter 17, Springer, Berlin, 2008, 391-410.

2. Segway Personal Transporters. Bedford, NH, 2012.

http://www.segway.com.
3. Yamamoto, Y. NXTway-GS (Self-Balanced Two-Wheeled Robot Controller Design). http://www.mathworks.com/matlabcentral/ fileexchange/ .

4. Double Robotics. Sunnyvale, CA, 2014.

http://www.doublerobotics.com .

5. Chan, R. P. M., K. A. Stol, C. R. Halkyard. Review of Modelling and Control of Two-wheeled Robots.m - Annual Reviews in Control, 37, 2013, 89-103.

6. Goodwin, G. C., S. F. Graebe, M. E. Salgado. Control System Design, Ch. 10. Prentice Hall, Upper Saddle River, NJ, 2001, ISBN: 9780139586538.

7. Ljung, L. System Identification: Theory for the User, 2nd Ed. Prentice-Hall, Inc., Englewood Cliffs, NJ, 1999, ISBN: 9780136566953.

8. Slavov, Ts., P. Petkov. Strapdown Inertial System Based on Improved MEMS Error Models. - Cybernetics and Information Technologies, 11, 2011, 3-23.

9. Zhou, K., J. C. Doyle, K. Glover. Robust and Optimal Control. Prentice Hall, Upper Saddle River, NJ, 1996, ISBN: 0-13-456567-3.

10. Spectrum Digital, Inc. 2007. eZdspTMF28335 Technical Reference. http://c2000.spectrumdigital.com/ezf28335/docs/ ezdspf28335c techref.pdf. 


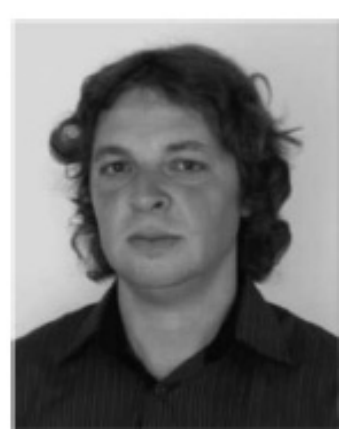

Tsonyo Slavov was born in Sofia, Bulgaria in 1978. He graduated from the Department of Systems and Control of the Technical University of Sofia in 2002. He received Ph.D degree in 2007. Presently he is an Associated Professor in the Department of Systems and Control at the Technical University of Sofia.

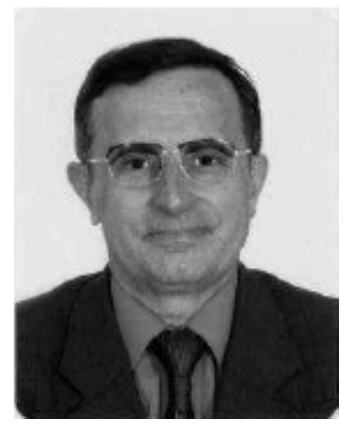

Petko Petkov received M.S. and Ph.D. degrees in control engineering from the Technical University of Sofia in 1971 and 1979. Since 1995, he has been a Professor of Control Theory in the Department of Systems and Control at the Technical University of Sofia. He is coauthoring Computational Methods for Linear Control Systems (Prentice Hall, Hemel Hempstead, U.K., 1991), Perturbation Theory for Matrix Equations (North-Holland, Amsterdam, 2003), and Robust Control Design with MATLAB (Springer, London, 2005, 2013).

Contacts:

Department of Systems and Control Technical University of Sofia 1756 Sofia, Bulgaria Corresponding author, e-mail: ts_slavov@tu-sofia.bg

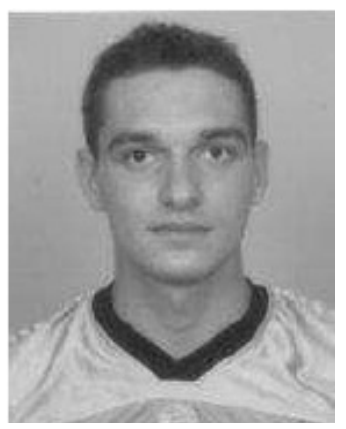

Jordan Kralev has M.S. degree in Automatics, Information and Control Technologies. Since 03.2013 he is a Ph.D. student in the Department of Systems and Control, Technical University of Sofia. His work is in the field of implementation of complex control structures with programmable platforms. 